\title{
Lesiones por armas de fuego desde la perspectiva médico-criminalística*
}

\author{
Drs. GABRIEL GARCÍA P. ${ }^{1,2,3}$, FERNANDA DEICHLER V. ${ }^{3,4}$, ESTEBAN TORRES E. . $^{4,5}$ \\ 1 Departamento de Cirugía, Pontificia Universidad Católica de Chile. \\ 2 Departamento de Medicina Criminalística de la Policía de Investigaciones de Chile. \\ 3 Servicio de Urgencia del Complejo Asistencial Dr. Sótero del Río. \\ 4 Equipo Cirugía Plástica, Hospital Dr. Sótero del Río. \\ 5 Departamento Cirugía, Pontificia Universidad Católica. \\ Santiago, Chile.
}

\section{Gunshoth wounds. Forensic considerations}

Dentro de las causas de muerte, los homicidios han tenido un aumento importante en los últimos años, siendo las lesiones por armas de fuego responsables de casi de un tercio. Por esto, es cada vez más frecuente que los cirujanos de los servicios de urgencia nos veamos enfrentados a trauma por armas de fuego. Conocer el trayecto probable del proyectil, la posibilidad de lesión concomitante por gases, sospechar la presencia de un taco dentro de la herida, etc, nos puede orientar en la toma de decisiones tanto antes como dentro del pabellón. El objetivo de esta revisión es proporcionar las nociones básicas de las características de estas lesiones para su correcta interpretación desde la óptica de la patología quirúrgica, y para describirlas y conservarlas lo mejor posible desde el punto de vista de la patología forense.

Chile tiene una tasa de mortalidad por homicidios que ubica al país en el quinto lugar en América Latina $^{1}$. Es preocupante constatar que las tasas han aumentado significativamente en años recientes. Las causas más frecuentes de muerte son las lesiones por arma blanca (39\%) y de fuego (28\%)․ La mayor incidencia de heridas por armas de fuego se produce en barrios de nivel social bajo, los fines de semana y por armas cortas.
Cada vez es más frecuente que los cirujanos nos veamos enfrentados a trauma por armas de fuego, por lo que es importante tener nociones básicas de las características de este tipo de lesiones y sus implicancias médico legales. Además del valor criminalístico y forense, reconocer algunas características de las lesiones por armas de fuego nos permite anticipar las lesiones que encontraremos en el pabellón y planificar la intervención que realizaremos. Siendo los proyectiles radiopacos, el rol de la radiología es indiscutido².

Las lesiones por arma de fuego se definen como el conjunto de alteraciones producidas en el organismo por el efecto de los elementos que integran el disparo en las armas de fuego. Por su parte, las armas de fuego se definen como aquellos instrumentos destinados a lanzar violentamente ciertos proyectiles aprovechando la fuerza expansiva de los gases que se producen en su interior por deflagración de la pólvora. Estos proyectiles poseen una gran energía cinética o fuerza remanente por lo que alcanzan largas distancias con gran capacidad de penetración ${ }^{3}$. Sólo como una aproximación mencionaremos que los proyectiles de armas cortas poseen velocidades de alrededor de 350 metros por segundo $(\mathrm{m} / \mathrm{s})$ y las armas largas, de alrededor de $1.000 \mathrm{~m} / \mathrm{s}$.

*Recibido el 16 septiembre de 2010 y aceptado para publicación el 12 de diciembre de 2010.

Correspondencia: Dr. Gabriel García P.

Estrella Solitaria 5153, Santiago, Chile. ggarcia@med.puc.cl 
Desde el punto de vista médico-quirúrgico, las heridas por arma de fuego se clasifican entre las contusas y se describen como contusiones simples con solución de continuidad. En las lesiones por armas de fuego se pueden distinguir tres componentes: orificio de entrada, trayectoria y orificio de salida ${ }^{3}$.

\section{Orificio de entrada}

El orificio de entrada corresponde a una herida contusa; sus características dependerán del tamaño de la munición, si está deformada por un impacto previo, si es proyectil único o múltiple, el ángulo de incidencia, si atravesó la ropa, etc ${ }^{3}$.

Se produce por el impacto del proyectil en la piel donde la presión ejercida supera la resistencia de la dermis. Es un orificio forzado a través de un tejido elástico, la dermis, lo cual explica que el orificio de entrada sea de menor diámetro que el proyectil que lo generó, por lo que no puede inferirse el calibre a partir de éste ${ }^{2,3}$.

El orificio de entrada esta conformado por los denominados "elementos constantes": el anillo de limpieza, el anillo contuso erosivo y la infiltración sanguínea. Además de otros elementos que no son constantes: el halo carbonoso, el tatuaje y la quemadura. La presencia de los segundos dependerá de la distancia del disparo y si hay interposición de ropa u otros elementos entre el arma y la piel de la víctima ${ }^{6}$.

El anillo de limpieza es el primero de adentro hacia afuera; se produce porque el material que va agregado a la superficie del proyectil (restos de lubricante, partículas metálicas, productos de la deflagración de la pólvora, restos de tela, etc) queda retenido en la zona más angosta del cono de presión ${ }^{4}$. Es

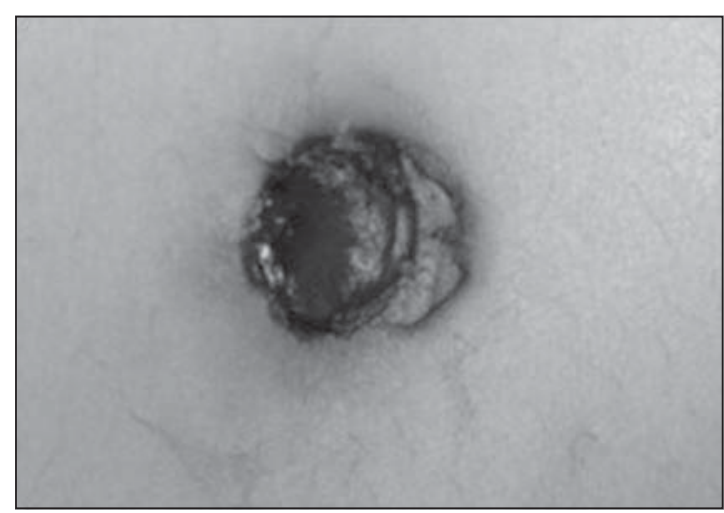

Figura 1. Anillo de limpieza, anillo contuso erosivo e infiltración sanguínea. menos evidente cuando el proyectil atraviesa ropa u otros elementos donde un porcentaje del material de superficie queda retenidos en ellos ${ }^{3}$.

El anillo contuso erosivo corresponde a una zona de dermis expuesta; producto del roce del proyectil contra la piel determinando la pérdida de la epidermis en el cono de presión. Es el segundo de adentro hacia fuera; su forma y simetría dependerá del ángulo de incidencia del proyectil respecto de la piel, lo cual nos orientará respecto de la trayectoria probable dentro del cuerpo (Figura 1$)^{5}$.

El trauma causado a los tejidos por el proyectil determina una infiltración sanguínea periférica al orificio de entrada, por ruptura de los vasos capilares de la dermis. La condición necesaria para que se produzca esto es que exista circulación de la sangre al momento del disparo, es decir, que la víctima haya estado con vida ${ }^{6}$.

El halo carbonoso (falso tatuaje o ahumamiento) corresponde fundamentalmente al depósito de los elementos de deflagración de la pólvora alrededor del orificio de entrada (Figura 2). Es susceptible de ser removido con el aseo de la piel. Puede quedar retenido parcial o totalmente por las vestimentas de la víctima. Si la distancia del disparo es mínima, o con apoyo del cañón contra la piel, no se producirá, ya que el material que lo compone ingresará a través del orificio de entrada hacia los planos profundos, pudiendo verse, ocasionalmente, en el interior de la herida ${ }^{2}$. Si la distancia es demasiado grande, los elementos de la deflagración se dispersarán en el aire, por lo que no existirá el halo ${ }^{3}$.

El tatuaje se produce por la incrustación, en la piel, de granos de pólvora incompletamente combustionados y partículas metálicas (Figura 3$)^{2}$. Al quedar incrustados en el espesor de la piel, no son susceptibles de ser removidos con en lavado de ésta.

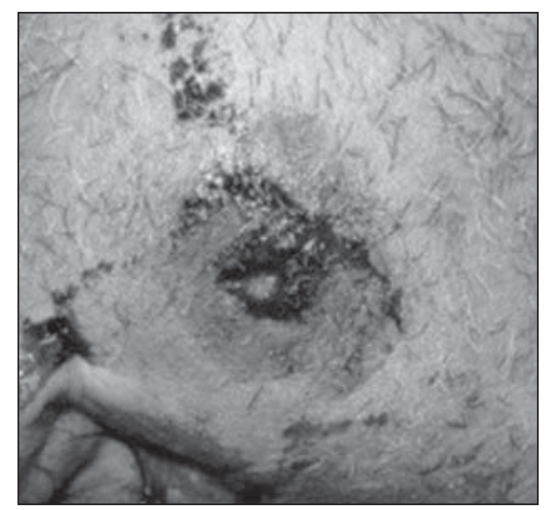

Figura 2. Halo carbonoso. 


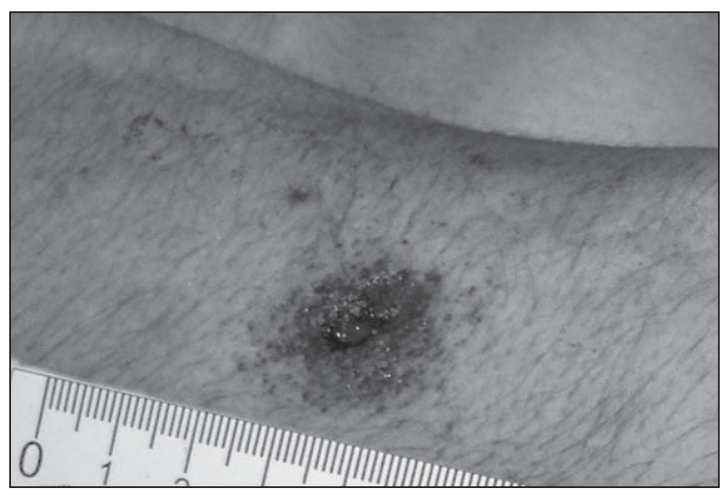

Figura 3. Tatuaje.

Rigen las mismas consideraciones que para el halo carbonoso respecto de la distancia del disparo . $^{\circ}$

El fogonazo que sale por la boca del cañón producirá una quemadura; su fugacidad impide que sea relevante en la piel. Puede incluir piel, vello y cabello. También puede incluir estructuras profundas si el disparo ha sido hecho a corta distancia, y la vestimenta que estuviere interpuesta entre este y la piel 6 .

Cuando el disparo es realizado a corta distancia o con apoyo del cañón, es importante considerar si es una zona donde la piel se encuentra sobre un plano óseo, ya que se presentarán características especiales: lesión de Hoffman, lesión estrellada, signo de Benassi e impresión del cañón en la piel.

El "cuarto de mina" o lesión de Hoffman corresponde a un bolsillo entre la piel y el hueso; producido por la expansión brusca del chorro de gas comprimido posterior al disparo ${ }^{6}$.

La falta de distensibilidad del plano óseo causa una salida explosiva de los gases hacia el exterior por el orificio de entrada, produciendo desgarros radiales de los bordes de la piel, resultando una lesión “estrellada” (Figura 4) (2,3,6. $^{2}$

El signo de Benassi corresponde al halo carbonoso en el orificio de entrada en el hueso.

La impresión del cañón en la piel (Figura 5) se produce en las zonas donde la piel no descansa sobre

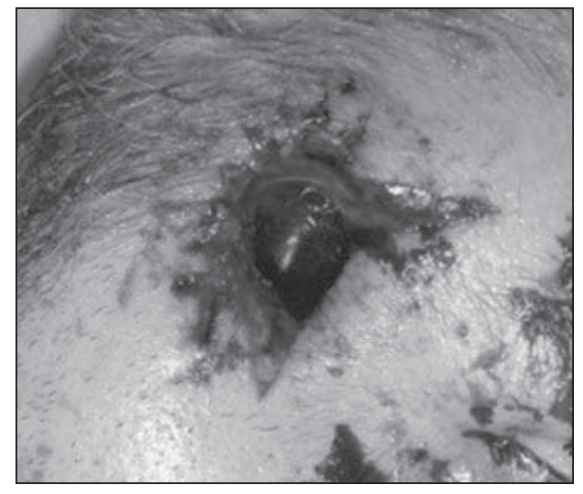

Figura 4. Lesión estrellada.

un plano rígido y la expansión brusca de los gases en el plano subcutáneo causará un abombamiento de esta hacia afuera, comprimiéndola contra el cañón ${ }^{2,3}$.

\section{Trayectoria intracorporal}

El trayecto del proyectil al interior del cuerpo puede ser rectilíneo o desviarse al chocar con huesos, por lo que al realizar el examen clínico, considerando la ubicación de los orificios de entrada y salida y otros hallazgos del examen físico, sólo puede presumirse la trayectoria ${ }^{6}$.

En lesiones por múltiples proyectiles, especialmente si estos se concentran en un segmento corporal puede resultar particularmente difícil correlacionar los orificios de entrada con los de salida y establecer las trayectorias de cada uno de estos.

Una vez que el proyectil ha penetrado en el organismo, la forma que tenga o adquiera, la energía cinética que posea y las estructuras que encuentre a su paso determinarán su trayectoria y las lesiones que cause, así como si saldrá o permanecerá dentro del cuerpo ${ }^{3}$.

Durante su trayecto intracorporal el proyectil libera energía cinética hacia los tejidos circundantes en forma perpendicular a su trayectoria, generando

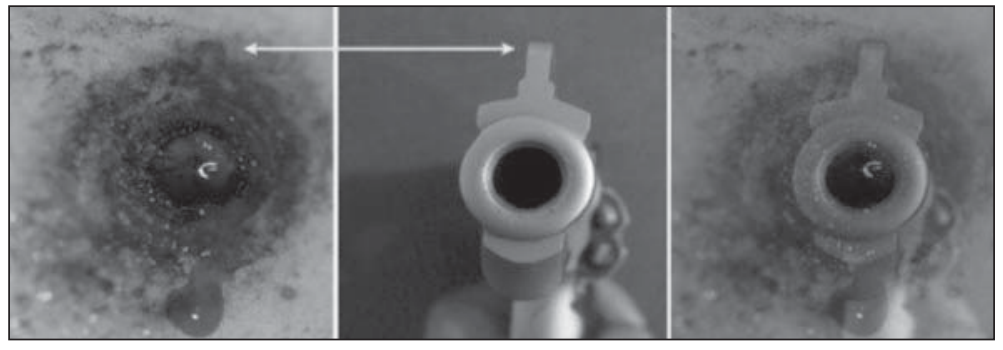

Figura 5. Impresión del cañón en la piel. 
una onda de choque que, debido a la elasticidad de estos, produce un espacio denominado "cavidad temporal”. Una vez disipada la energía, las estructuras retornan a su ubicación, quedando sólo el trayecto del proyectil; pero si la onda expansiva supera la resistencia de los tejidos se produce una ruptura de estos, generando una cavidad definitiva mayor que el diámetro del proyectil ${ }^{2,3}$. Esto es particularmente significativo en las vísceras macizas, como el hígado o el bazo, pero reviste poca importancia en las de baja densidad, como el pulmón ${ }^{2}$.

Asimismo, la onda expansiva de los proyectiles de alta velocidad puede generar daño a distancia en los vasos sanguíneos, produciendo lesión de la íntima y el endotelio vascular, y secundariamente trombosis e isquemia distal, sin que exista lesión visible del tejido ${ }^{3,6}$.

El proyectil puede desviar su trayectoria al atravesar o golpear estructuras de distinta densidad, lo que se puede traducir en una trayectoria distinta de la rectilínea ${ }^{5}$. Si bien es cierto que al impactar contra un hueso tanto éste como el proyectil pueden fragmentarse, la pérdida de energía cinética es tal que los fragmentos resultantes tienen escasa capacidad de dañar tejidos ${ }^{2}$.

Las lesiones que puede causar un proyectil incluyen todos los órganos y estructuras. Sin embargo, merecen especial mención las siguientes:

- Cráneo: la cavidad craneana es una bóveda ósea inextensible, por lo que la onda de choque produce un aumento de la presión intracraneana, con el consiguiente daño al tejido encefálico y la salida de parte de éste por el orificio de entrada y de salida. Si la energía cinética de la onda de choque supera la resistencia de las suturas de los huesos del cráneo, entonces se produce un estallido de este, el cual es perceptible al examinar el cadáver ${ }^{2}$.

- Vísceras macizas: en órganos macizos cuyo tejido presenta escasa elasticidad, como el hígado o el bazo, cuando la energía de la onda de choque supera su resistencia se produce el estallido de parte o la totalidad del órgano ${ }^{2}$.

- Dentro del organismo el proyectil o sus fragmentos pueden desplazarse y embolizar, con mayor frecuencia, en el sistema arterial ${ }^{2}$. En la experiencia de uno de los autores un proyectil calibre 6.25 mm obstruyó la uretra y finalmente fue expulsado a través de ésta.

- En los disparos con apoyo de cañón o a corta distancia, el gas producido por la deflagración de la pólvora penetra en el orificio de entrada para luego expandirse dentro de los tejidos. Este hecho reviste importancia en los compartimentos esca- samente distensibles como el cráneo o la mano, donde el daño tisular que produce puede incluso ser de mayor magnitud que el del proyectil; o en el caso de las escopetas y rifles, cuyos cartuchos generan una gran cantidad de gas ${ }^{2}$.

Los proyectiles no tienen indicación de ser extraídos sólo por encontrarse dentro del cuerpo, ya que el procedimiento no está exento de riesgo y los casos de intoxicación por plomo descritos en la literatura son escasos. Sin embargo, si se encuentra un proyectil fácilmente accesible, éste debe ser retirado, minimizando el daño en la superficie que presenta la impresión de las estrías del cañón que la disparó, ya que éstas son únicas para cada arma y permiten, mediante un estudio comparativo, identificarla ${ }^{1}$. Debe intentarse extraer el proyectil digitalmente. Si esto no es posible puede tomarse firmemente con una pinza Kelly, evitando que sus mandíbulas resbalen sobre la superficie. El proyectil constituye una evidencia y debe ser manejado con la cadena de custodia respectiva.

\section{Orificio de salida}

Se forma por la presión ejercida por el proyectil desde dentro hacia afuera, evertiendo la piel, por lo que no presenta las características del orificio de entrada (anillo de limpieza ni contuso-erosivo), sin embargo, puede presentar un borde erosivo que se genera por el contacto de la epidermis evertida con la ropa u otra superficie ${ }^{2}$. Puede dar salida a restos de los órganos lesionados en el trayecto.

Requiere que el proyectil conserve suficiente energía cinética tras su paso por el cuerpo para vencer la resistencia de la dermis. Habitualmente son de tamaño similar al de entrada, pero, puede ser de mayor tamaño en proyectiles de alta velocidad o que se presenten expandidos (proyectiles diseñados para expandirse) o deformados (por impactos dentro o fuera del cuerpo) $)^{2}$.

Debe evitarse suturar los orificios de entrada y salida, salvo que sea estrictamente necesario, así como incluirlos en la herida operatoria o utilizarlos para instalar drenajes, ya que entregan información que puede ser extremadamente útil desde el punto de vista criminalístico ${ }^{2}$.

\section{Heridas por perdigones}

Cada uno de los perdigones es un proyectil, y va a dar lugar a un orificio de entrada y un trayecto independiente. El tamaño del área de dispersión dependerá de la distancia a la que se ha efectuado 


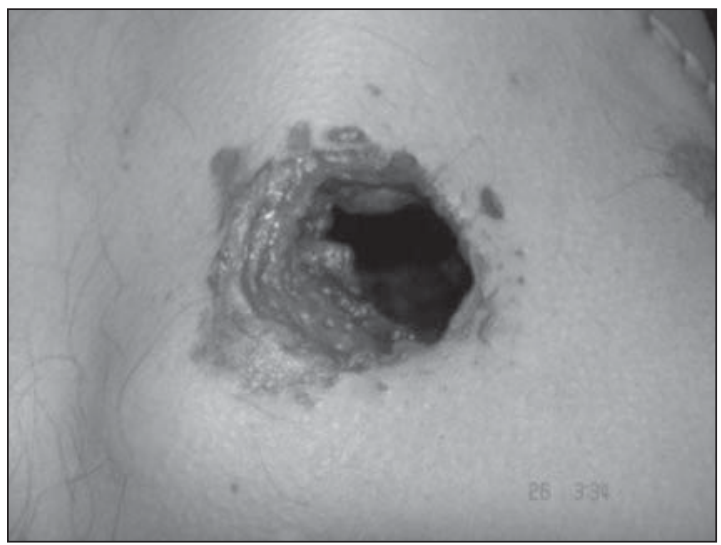

Figura 6. Herida por perdigones de corta distancia.

el disparo. Cuando la distancia entre la boca del arma de fuego y el cuerpo es escasa, no se produce dispersión de los perdigones, así todo el conjunto se traslada como una sola masa y produce una gran herida de morfología irregular con bordes en forma de sacabocados, que semeja un orificio horadado por una rata (rat hole) y que, a diferencia de los orificios de bala, sí tiene relación directa con el diámetro del cañón (Figura 6). Estas lesiones producen gran destrucción tisular y tienen una alta mortalidad ${ }^{3,6}$.

A mayor distancia la dispersión de los proyectiles (perdigones) y sus respectivos orificios de entrada será mayor y su capacidad de penetración, menor, ya que poseen menor energía cinética. No es infrecuente que no existan orificios de salida ya que estos proyectiles, de poca masa, pierden su energía cinética rápidamente en el interior del organismo. Hay que tener presente que en disparos de corta distancia el taco, o pistón, que impulsa a los perdigones puede

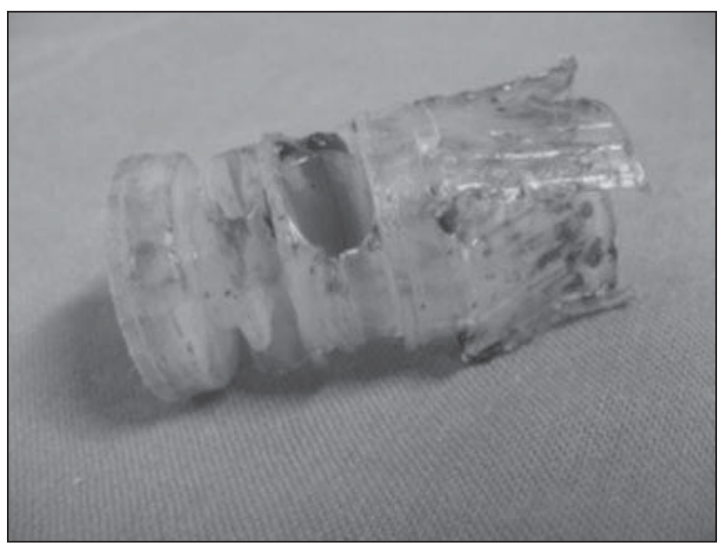

Figura 7. Taco extraído desde una herida por escopeta.

entrar en la herida producida por éstos, por lo que debe ser buscado dirigidamente (Figura 7$)^{3}$.

\section{Referencias}

1. Medina E, Kaempffer AM. Consideraciones epidemiológicas sobre los traumatismos en Chile. Rev Chil Cir. 2007;59:175-84.

2. Di Maio, Vincent JM. Gunshot Wounds - Practical Aspects of Firearms Ballistics and Forensic Techniques. CRC Press, U.S.A., 1999.

3. Dood, Malcom J. Terminal Ballistic - A Text and Atlas of Gunshoth Wounds, CRC Press, U.S.A., 2006.

4. Ferreyro MF. Balística Manual, Editorial B de F, Uruguay-Argentina, 2007.

5. Fisher C, Barry AJ. Techniques of Crimen Scene Investigation, CRC Press, U.S.A., 2004.

6. Teke A. Medicina Legal y Criminalística, Ediciones Jurídicas de Santiago, Chile, 2005. 